\title{
Explicit formulae for one-part double Hurwitz numbers with completed 3-cycles
}

\author{
Viet Anh Nguyen ${ }^{1}$
}

Received: 14 November 2016 / Accepted: 28 July 2018 / Published online: 12 August 2018

(c) Springer Science+Business Media, LLC, part of Springer Nature 2018

\begin{abstract}
We prove two explicit formulae for one-part double Hurwitz numbers with completed 3-cycles. We define "combinatorial Hodge integrals" from these numbers in the spirit of the celebrated ELSV formula. The obtained results imply some explicit formulae and properties of the combinatorial Hodge integrals.
\end{abstract}

Keywords Hurwitz numbers · Symmetric groups $\cdot$ Symmetric functions

\section{Introduction}

\subsection{Notation}

First of all, let us set up some notations. For a natural number $d$, a partition $\mu$ of $d$, denoted by $\mu \vdash d$, is a sequence of non-increasing strictly positive integers $\mu=$ $\left(\mu_{1} \geq \mu_{2} \geq \cdots \geq \mu_{m}\right)$ such that $d=\mu_{1}+\cdots+\mu_{m}$. We call $m$ the length of $\mu$, denoted by $l(\mu)$. Another way of writing $\mu$ is $\mu=\left(1^{m_{1}}, 2^{m_{2}}, \ldots\right)$ which tells us that $i$ appear $m_{i}$ times. Define $|\operatorname{Aut}(\mu)|:=m_{1} ! m_{2} ! \ldots$ If $m_{i}=0$, we can choose to write $i$ or not depending on which choice is convenient in each context. We reserve the Greek letters for partitions. The set of all partitions of $d$ is denoted by $\operatorname{Part}(d)$, and the set of all partitions is denoted by Part.

For a series $f(z)=\sum a_{i} z^{i}$, we denote $\left[z^{i}\right] f:=a_{i}$.

\subsection{Hurwitz numbers}

Hurwitz numbers (in many variants) appeared at the cross-road of many active directions in contemporary mathematics and mathematical physics, such as the combinatorics of symmetric groups and graphs on surfaces, the intersection theory in

\footnotetext{
$\varangle \quad$ Viet Anh Nguyen

vnguyen@math.univ-angers.fr

1 LAREMA UMR CNRS 6093, Université d'Angers, Angers, France
} 
algebraic geometry, tau functions in integrable systems and tropical geometry (see, for instance $[2,6,8,10])$.

In their simplest version, given two positive integers $d$ and $g$, and a partition $\beta$ of $d$, connected (disconnected) single Hurwitz number ${ }^{1} H_{\beta}^{g}$ count the number of equivalence classes of branched $d$-coverings of the Riemann sphere by a connected (possibly disconnected) Riemann surface of genus $g$, assuming that one branch point has branching given by $\beta$ and all other branch points are simple. Some very interesting results connect these objects with other areas of mathematics. In particular, single Hurwitz numbers satisfy KP equations [11], Virasoro constraints [1] and ChekhovEynard-Orantin topological recursion [4].

Further interesting results have been proved and conjectured for other types of Hurwitz numbers. In this article, we are concerned with double Hurwitz numbers with completed cycles [13].

Actually, enumerating branched coverings is just one of the possible sources of Hurwitz numbers. It is known that, equivalently, Hurwitz numbers enumerate permutations with certain properties, ribbon graphs (i.e., graphs on Riemann surfaces) and tropical graphs (see again $[2,6,8,10]$ and references therein). In particular, the interpretation in terms of permutations leads to the combinatorial definition of Hurwitz numbers in terms of irreducible characters of the symmetric groups.

In this paper, as it is done in [13], we study Hurwitz numbers starting from a purely combinatorial definition. Our results are about one-part double Hurwitz numbers with completed 3-cycles $H_{(d), \beta}^{g,(2)}$ and can be seen as a natural analogue of some well known results by Goulden et al. [6] about standard double Hurwitz numbers $H_{\alpha, \beta}^{g}$. They found the following explicit formulas for one-part double Hurwitz numbers (in this case connected $=$ disconnected)

Theorem 1.1 [6] Let $g \geq 0$ and $\beta \vdash d$ a partition of $d$. Then

$$
\begin{aligned}
H_{(d), \beta}^{g} & =k ! d^{k-1}\left[z^{2 g}\right] \prod_{j \geq 1}\left(\frac{\sinh (j z / 2)}{j z / 2}\right)^{c_{j}} \\
& =\frac{k ! d^{k-1}}{2^{2 g}} \sum_{\lambda \vdash g} \frac{\xi_{2 \lambda} S_{2 \lambda}}{|\operatorname{Aut}(\lambda)|} .
\end{aligned}
$$

The notation will be explained later. The analogues for one-part double Hurwitz numbers (again, connected $=$ disconnected in this case) with completed 3-cycles, proved in this article, are

Theorem 1.2 Given $g \geq 0, d>0$, let $\beta$ be a partition of odd length of $d$ and $s$ be an integer such that $2 s=2 g-1+l(\beta)$. Then we have:

\footnotetext{
1 The Riemann-Hurwitz theorem relate $d, g$ and $\beta$.
} 


$$
\begin{aligned}
H_{(d), \beta}^{g,(2)} & =\frac{s ! d^{s-1}}{2^{s}} \sum_{h=0}^{g} \frac{(2 s-2 h) !}{h !(s-h) ! 12^{h}} d^{2 h}\left[z^{2(g-h)}\right] \prod_{i \geq 1}\left(\frac{\sinh (i z / 2)}{i z / 2}\right)^{c_{i}} \\
& =\frac{s ! d^{s-1}}{2^{s+2 g}} \sum_{h=0}^{g} \frac{(2 s-2 h) !}{h !(s-h) ! 3^{h}} d^{2 h} \sum_{\lambda \vdash(g-h)} \frac{\xi_{2 \lambda} S_{2 \lambda}}{|A u t(\lambda)|}
\end{aligned}
$$

The reason why our method does not work for $H_{(d), \beta}^{g,(r)}$ with $r>2$ is explained in the remark after Eq. (13).

Let us mention that Hurwitz numbers with completed cycles were shown to be relative Gromov-Witten invariants of $\mathbb{P}^{1}$ (under some normalization) [12]. It is also conjectured that there should be an ELSV-like formula for one-part double Hurwitz numbers with completed cycles [13]. From this point of view, we believe that it is of a great use to have an efficient formula for computing these numbers.

To conclude, let us summarize some interesting consequences of the explicit formulae above. First, as it is in the case of standard double Hurwitz numbers, $H_{(d), \beta}^{g,(2)}$ is polynomial in the parts of $\beta$ (for fixed $g$ and $l(\beta)$ ), with the highest and lowest degrees being respectively $3 g+\frac{l(\beta)-3}{2}$ and $g+\frac{l(\beta)-3}{2}$. Note that the so-called strong piecewise polynomiality is proven in [13] for all double Hurwitz numbers with completed cycles. For one-part numbers, piecewise polynomiality becomes polynomiality. Thus our formula should be viewed as an illustration of this fact through an explicitly computable case.

Second, single Hurwitz numbers are connected, through the celebrated EkedahlLando-Shapiro-Vainshtein (ELSV) formula, to Hodge integrals on the DeligneMumford moduli space of stable curves with marked points [3]. A similar connection between double Hurwitz numbers and integrals of cohomological classes over a (yet to be defined) moduli space is conjectured in [6] (see also [13] for a similar conjecture for double Hurwitz numbers with completed cycles). Following what is done in [6] to support their conjecture, we defined and study "combinatorial Hodge integrals" [Eq. (24)]. We prove that the lowest degree Hodge integrals satisfy a formula (Proposition 4.2) which is an analogue of the $\lambda_{g}$-theorem by Faber and Pandharipande [5]. It is also an analogue of [6, Prop. 3.12].

Moreover, these lowest degree combinatorial Hodge integrals, satisfy a (modified) version of the string and dilaton equations. Assembling them in a generating function $F$, we prove that the string and dilaton equations correspond to two linear operators $L_{-1}$ and $L_{0}$ annihilating $F$ and satisfying a Virasoro-like relation. It would be of great interest to prove that a whole set of Virasoro-like constraints can be obtained.

Finally, we prove a closed formula for the combinatorial Hodge integrals of top degree.

The paper is organized as follows. Section 2 is devoted to the combinatorial definition of double Hurwitz numbers with completed cycles. Afterward, in Sect. 3, we state and prove our main result, Theorem 1.2. The corollaries are stated and proved in the last section. 


\section{Double Hurwitz numbers with completed cycles}

We follow closely the exposition in [13] that gives a combinatorial definition of double Hurwitz numbers with completed cycles.

\subsection{Shifted symmetric functions}

Let $\mathbb{Q}\left[x_{1}, \ldots, x_{d}\right]$ be the algebra of $d$-variable polynomials over $\mathbb{Q}$. The shifted action of the symmetric group $S_{d}$ (the group of permutations on $\{1, \ldots, d\}$ ) on this algebra is defined by:

$$
\sigma\left(f\left(x_{1}-1, \ldots, x_{d}-d\right)\right):=f\left(x_{\sigma(1)}-\sigma(1), \ldots, x_{\sigma(d)}-\sigma(d)\right)
$$

for $\sigma \in S_{d}$ and for any polynomial written in the variables $x_{i}-i$. Denote by $\mathbb{Q}\left[x_{1}, \ldots, x_{d}\right]^{\star}$ the subalgebra of polynomials which are invariant with respect to this action.

Define the algebra of shifted symmetric functions as the projective limit

$$
\Lambda^{\star}:=\lim _{\longleftarrow} \mathbb{Q}\left[x_{1}, \ldots, x_{d}\right]^{\star},
$$

where the projective limit is taken in the category of filtered algebras with respect to the homomorphism which sends the last variable to 0 . Concretely, an element of this algebra is a sequence $f=\left\{f^{(d)}\right\}_{d \geq 1}, f^{(d)} \in \mathbb{Q}\left[x_{1}, \ldots, x_{d}\right]^{\star}$ such that the polynomials $f^{(d)}$ are of uniformly bounded degree and stable under the restriction $\left.f^{(d+1)}\right|_{x_{d+1}=0}=f^{(d)}$.

\subsection{Bases of the algebra of shifted symmetric functions}

Definition 2.1 For any positive integer $k$, define the corresponding shifted symmetric power sum:

$$
p_{k}\left(x_{1}, x_{2}, \ldots\right):=\sum_{i=1}^{\infty}\left(\left(x_{i}-i+\frac{1}{2}\right)^{k}-\left(-i+\frac{1}{2}\right)^{k}\right)
$$

In the following, we are only interested in evaluating these functions on partitions. That is, for a partition $\lambda=\left(\lambda_{1} \geq \lambda_{2} \geq \ldots\right)$, we define $p_{k}(\lambda):=p_{k}\left(\lambda_{1}, \lambda_{2}, \ldots\right)$. As usual in symmetric function theory, for any partition $\mu$, define $p_{\mu}=p_{\mu_{1}} p_{\mu_{2}} \ldots$

The functions $\left\{p_{\mu}, \mu \in\right.$ Part $\}$ form a basis of $\Lambda^{\star}$. Another basis is defined as follows. For partitions $\lambda$ and $\mu$ of $d$, let $\chi_{\mu}^{\lambda}$ be the irreducible character of $S_{d}$ associated with $\lambda$ evaluated at $\mu$ (or more precisely, at a permutation of type $\mu$ ), $\operatorname{dim} \lambda=\chi_{\left(1^{n}\right)}^{\lambda}$ be the dimension of the irreducible representation, and $\operatorname{Per}(\mu)$ be the set of permutations of $S_{n}$ whose cycle structure is described by $\mu$. 
Definition 2.2 For two partitions $\lambda$ and $\mu$ of $d$, define

$$
f_{\mu}(\lambda):=|\operatorname{Per}(\mu)| \frac{\chi_{\mu}^{\lambda}}{\operatorname{dim}(\lambda)} .
$$

Kerov and Olshanski [9] proved that:

Proposition 2.1 The functions $\left\{f_{\mu}, \mu \in\right.$ Part $\}$ are shifted symmetric functions, and form a basis of $\Lambda^{\star}$.

\subsection{Completed cycles}

Let $\mathbb{Q} S_{d}$ be the group algebra of $S_{d}$ over $\mathbb{Q}$, i.e., the algebra of formal linear sum with rational coefficients of elements of $S_{d}$. Let $Z \mathbb{Q} S_{d}$ be the center of this algebra, which is the subalgebra containing elements that commute with every element of $\mathbb{Q} S_{d}$. Finally, define

$$
Z:=\bigoplus_{d=0}^{\infty} Z \mathbb{Q} S_{d}
$$

It is well known that a basis of $Z$ can be constructed as follows. For a partition $\mu$, let

$$
C_{\mu}:=\sum_{g \in \operatorname{Per}(\mu)} g .
$$

Then $\left\{C_{\mu}, \mu \in \mathbf{P a r t}\right\}$ form a basis of $Z$. Therefore we have the linear isomorphism

$$
\begin{aligned}
\phi: Z & \rightarrow \Lambda^{\star} \\
C_{\mu} & \mapsto f_{\mu} .
\end{aligned}
$$

Definition 2.3 For any partition $\mu$, the completed $\mu$-conjugacy class $\bar{C}_{\mu}$ is defined as $\bar{C}_{\mu}:=\phi^{-1}\left(p_{\mu}\right) / \prod \mu_{i} !^{2}$ Of special interest are the completed cycles $\overline{(r)}:=\bar{C}_{(r)}, r \in$ N. ${ }^{3}$

\subsection{Double Hurwitz numbers with completed cycles}

From now on, we shall follow the notation of Goulden et al. [6], so that the readers can compare our result with theirs easily. Let $\alpha$ and $\beta$ be two partitions of a number $d$, whose lengths are $m$ and $n$ respectively. Let $g, r$ and $s$ be three nonnegative integers such that $r s=2 g-2+m+n$.

Definition 2.4 Disconnected double Hurwitz numbers with completed $(r+1)$-cycles are defined as:

\footnotetext{
2 This normalization, following [14], is different from that of [12].

${ }^{3}$ Recall that $(r)$ denotes the 1-part partition of $r$.
} 


$$
H_{\alpha, \beta}^{g,(r)}:=\frac{1}{\prod \alpha_{i} \prod \beta_{j}} \sum_{\lambda \vdash d} \chi_{\alpha}^{\lambda}\left(\frac{p_{r+1}(\lambda)}{(r+1) !}\right)^{s} \chi_{\beta}^{\lambda} .
$$

We often omit the superscript $(r)$ if it is fixed in advance. Since the completed 2cycle is equal to the ordinary 2-cycle, the numbers $H_{\alpha, \beta}^{g,(1)}$ are just the ordinary double Hurwitz numbers.

We are mostly interested in the dependence of $H_{\alpha, \beta}^{g,(r)}$ on (the parts of) $\alpha$ and $\beta$, given fixed $g, r, l(\alpha)$ and $l(\beta)$. The numbers obtained in the case $m=1$, i.e., $\alpha=(d)$ are called one-part double numbers. In this case, the sum is simplified a lot, and we can get an explicit and compact formula for $r=2$.

\subsection{Combinatorial interpretation}

In [14, Sec. 2.2], the authors give a combinatorial interpretation of single Hurwitz numbers with completed cycles. We can naturally generalize their construction for double numbers as follows. Let $\alpha$ and $\beta$ be two partitions of $d$. A $(g, r, \alpha, \beta)$-factorization $\operatorname{fac}(g, r, \alpha, \beta)$ is a factorization in $S_{d}$ of the following form:

$$
h_{1} \ldots h_{s} g_{1} g_{2}=1 \text {, }
$$

where $r s=2 g-2+l(\alpha)+l(\beta), g \in \mathbb{Z}_{+}, g_{1} \in \operatorname{Per}(\alpha), g_{2} \in \operatorname{Per}(\beta)$, and each $h_{i} \in S_{d}$ appears in $\overline{(r+1)}$ with a coefficient $c_{i} \neq 0$. The weight of this factorization is defined as

$$
w(\mathbf{f a c}):=\prod_{i=1}^{s} c_{i}
$$

Proposition 2.2 We have the following equality:

$$
\sum_{\text {fac } \in\{(g, r, \alpha, \beta)-\text { factorizations }\}} w(\text { fac })=\frac{d !}{|\operatorname{Aut}(\alpha)||A u t(\beta)|} H_{\alpha, \beta}^{g,(r)} .
$$

\section{Explicit formula for one-part double Hurwitz numbers with completed 3-cycles}

We now consider the case $r=2$. Let $\beta$ be a partition of $d$ of odd length $n$. Let $s=g+\frac{n-1}{2}$. We write $\beta$ in three ways, each of which is convenient in each specific context:

$$
\left(\beta_{1}, \beta_{2}, \ldots\right)=\left(1^{n_{1}} 2^{n_{2}} \ldots\right)=\left(\ell^{n_{\ell}} \ldots q^{n_{q}}\right) .
$$

Here $\ell$ and $q$ are the smallest and greatest numbers appearing in $\beta$. If a number $i$ does not appear in $\beta, n_{i}=0$. Let $c_{i}=n_{i}$ for $i \geq 2$ and $c_{1}=n_{1}-1$. We have $\sum_{i} c_{i}=n-1$ and $\sum i c_{i}=d-1$. 
We can easily compute that:

$$
p_{3}\left(\left(d-k, 1^{k}\right)\right)=\left(d-k-\frac{1}{2}\right)^{3}-\left(-k-\frac{1}{2}\right)^{3}=3 d\left(\left(k-\frac{d-1}{2}\right)^{2}+\frac{d^{2}}{12}\right) .
$$

Remark The fact that $p_{3}\left(\left(d-k, 1^{k}\right)\right)$ has the form $a(k+b)^{2}+c$, where $a, b, c$ do not depend on $k$ (but on $d$ of course) turns out to be crucial for our method. Unfortunately, $p_{r+1}\left(\left(d-k, 1^{k}\right)\right)$ for $r \geq 3$ do not have the form $a(k+b)^{r}+c$, so we do not get a compact formula by the same strategy.

Lemma 3.1 We have the following irreducible character evaluation:

$$
\begin{aligned}
\chi_{\beta}^{\left(d-k, 1^{k}\right)}= & (-1)^{k}\left[z^{k}\right]\left(1+z+\cdots+z^{\ell-1}\right)\left(1-z^{\ell}\right)^{n_{\ell}-1} \prod_{i \geq \ell+1}\left(1-z^{i}\right)^{n_{i}} \\
& =(-1)^{k}\left[z^{k}\right] \prod_{i \geq 1}\left(1-z^{i}\right)^{c_{i}} \\
& =(-1)^{k} \sum_{h=0}^{\ell-1} \sum_{j_{\ell}=0}^{n_{\ell}-1} \sum_{j_{\ell+1}=0}^{n_{\ell+1}} \cdots \sum_{j_{q}=0}^{n_{q}}(-1)^{\sum_{i \geq l} j_{i}}\left(\begin{array}{c}
n_{\ell}-1 \\
j_{\ell}
\end{array}\right)\left(\begin{array}{c}
n_{\ell+1} \\
j_{\ell+1}
\end{array}\right) \ldots \\
& \left(\begin{array}{c}
n_{q} \\
j_{q}
\end{array}\right) \delta_{k, h+\sum_{i=\ell}^{q} i j_{i} .}
\end{aligned}
$$

here $\delta_{x, y}:=1$ if $x=y$, and 0 otherwise. This lemma is well known and can be derived from the Murnaghan-Nakayama rule. See, for instance, [6, p. 59].

For $j \geq 1$, let $\xi_{2 j}=\left[x^{2 j}\right] \log (\sinh x / x)$ and

$$
S_{2 j}=\sum_{k \geq 1} k^{2 j} c_{k}=-1+\sum_{k \geq 1} k^{2 j} n_{k}=-1+\sum_{k \geq 1} \beta_{k}^{2 j},
$$

i.e., $S_{2 j}$ is a power sum for the partition, shifted by 1 . For a partition $\lambda$, let $\xi_{\lambda}=$ $\xi_{\lambda_{1}} \xi_{\lambda_{2}} \ldots$ and $S_{\lambda}=S_{\lambda_{1}} S_{\lambda_{2}} \ldots$ and $2 \lambda=\left(2 \lambda_{1}, 2 \lambda_{2}, \ldots\right)$.

Lemma 3.2 The following formula holds true:

$$
\left[z^{2 k}\right] \prod_{i \geq 1}\left(\frac{\sinh (i z / 2)}{i z / 2}\right)^{c_{i}}=2^{-2 k} \sum_{\lambda \vdash k} \frac{\xi_{2 \lambda} S_{2 \lambda}}{|\operatorname{Aut}(\lambda)|} .
$$

Proof We have

$$
\begin{aligned}
\prod_{i \geq 1}\left(\frac{\sinh (i x)}{i x}\right)^{c_{i}} & =\exp \left(\sum_{i \geq 1} c_{i} \sum_{j \geq 1} \xi_{2 j} i^{2 j} x^{2 j}\right)=\exp \left(\sum_{j \geq 1} \xi_{2 j} S_{2 j} x^{2 j}\right) \\
& =\sum_{\lambda} \frac{\xi_{2 \lambda} S_{2 \lambda}}{|\operatorname{Aut}(\lambda)|} x^{2|\lambda|}
\end{aligned}
$$


The proof is finished upon setting $x=z / 2$.

Lemma 3.3 Let $S_{p}(k, x):=\sum_{h=0}^{k-1}(h+x)^{p}$. Then

$$
S_{p}(k, x)=\left[\frac{z^{p}}{p !}\right] e^{x z}\left(1+e^{z}+\cdots+e^{(k-1) z}\right) .
$$

Proof Indeed,

$$
\sum_{p=0}^{\infty} S_{p}(k, x) \frac{z^{p}}{p !}=\sum_{h=0}^{k-1} e^{z(h+x)}=e^{z x}\left(1+e^{z}+\cdots+e^{(k-1) z}\right) .
$$

We can now prove our main Theorem:

Theorem 1.2 Given $g \geq 0, d>0$, let $\beta$ be a partition of odd length of $d$ and $s$ be an integer such that $2 s=2 g-1+l(\beta)$. Then we have:

$$
\begin{aligned}
H_{(d), \beta}^{g,(2)} & =\frac{s ! d^{s-1}}{2^{s}} \sum_{h=0}^{g} \frac{(2 s-2 h) !}{h !(s-h) ! 12^{h}} d^{2 h}\left[z^{2(g-h)}\right] \prod_{i \geq 1}\left(\frac{\sinh (i z / 2)}{i z / 2}\right)^{c_{i}} \\
& =\frac{s ! d^{s-1}}{2^{s+2 g}} \sum_{h=0}^{g} \frac{(2 s-2 h) !}{h !(s-h) ! 3^{h}} d^{2 h} \sum_{\lambda \vdash(g-h)} \frac{\xi_{2 \lambda} S_{2 \lambda}}{|A u t(\lambda)|}
\end{aligned}
$$

Proof By definition:

$$
H_{(d), \beta}^{g,(2)}=\frac{1}{d \prod \beta_{j}} \sum_{\lambda \vdash d} \chi_{(d)}^{\lambda}\left(\frac{p_{3}(\lambda)}{6}\right)^{s} \chi_{\beta}^{\lambda}
$$

It is well known that $\chi_{(d)}^{\lambda}=0$ except for $\lambda=\left(d-k, 1^{k}\right), k=0, \ldots, d-1$, in which case it is equal to $(-1)^{k}$. So we have:

$$
\begin{aligned}
H_{(d), \beta}^{g,(2)} & =\frac{d^{s-1}}{2^{s} \prod \beta_{j}} \sum_{k=0}^{d-1}\left(\left(k-\frac{d-1}{2}\right)^{2}+\frac{d^{2}}{12}\right)^{s}(-1)^{k} \chi_{\beta}^{\left(d-k, 1^{k}\right)} \\
& =\frac{d^{s-1}}{2^{s} \prod \beta_{j}}\left[\frac{t^{s}}{s !}\right] \sum_{k=0}^{d-1} \exp \left\{t\left(\left(k-\frac{d-1}{2}\right)^{2}+\frac{d^{2}}{12}\right)\right\}(-1)^{k} \chi_{\beta}^{\left(d-k, 1^{k}\right)} \\
& =\frac{s ! d^{s-1}}{2^{s} \prod \beta_{j}}\left[t^{s}\right] \exp \left(\frac{t d^{2}}{12}\right) \sum_{k=0}^{d-1} \exp \left\{t\left(k-\frac{d-1}{2}\right)^{2}\right\}(-1)^{k} \chi_{\beta}^{\left(d-k, 1^{k}\right)} .
\end{aligned}
$$


We treat the sum separately now:

$$
\begin{aligned}
A= & \sum_{k=0}^{d-1} \exp \left\{t\left(k-\frac{d-1}{2}\right)^{2}\right\}(-1)^{k} \chi_{\beta}^{\left(d-k, 1^{k}\right)} \\
= & \sum_{h=0}^{\ell-1} \sum_{j_{\ell}=0}^{n_{\ell}-1} \sum_{j_{\ell+1}=0}^{n_{\ell+1}} \ldots \sum_{j_{q}=0}^{n_{q}} \exp \left\{t\left(h+\sum_{i \geq \ell} i j_{i}-\frac{d-1}{2}\right)^{2}\right\} \\
& \times(-1)^{\sum_{i \geq 1} j_{i}}\left(\begin{array}{c}
n_{\ell}-1 \\
j_{\ell}
\end{array}\right)\left(\begin{array}{c}
n_{\ell+1} \\
j_{\ell+1}
\end{array}\right) \ldots\left(\begin{array}{c}
n_{q} \\
j_{q}
\end{array}\right) . \quad \text { (Lemma3.1) }
\end{aligned}
$$

We expand the exponential and sum over $h$ first :

$$
\begin{aligned}
A= & \sum_{j_{\ell}=0}^{n_{l}-1} \sum_{j_{\ell+1}=0}^{n_{\ell+1}} \ldots \sum_{j_{q}=0}^{n_{q}}(-1)^{\sum_{i \geq \ell} j_{i}}\left(\begin{array}{c}
n_{\ell}-1 \\
j_{\ell}
\end{array}\right)\left(\begin{array}{c}
n_{\ell+1} \\
j_{\ell+1}
\end{array}\right) \ldots \\
& \left(\begin{array}{c}
n_{q} \\
j_{q}
\end{array}\right) \sum_{p=0}^{\infty} \frac{t^{p}}{p !} \sum_{h=0}^{\ell-1}\left(h+\sum_{i \geq \ell} i j_{i}-\frac{d-1}{2}\right)^{2 p} \\
= & \sum_{p=0}^{\infty} \frac{(2 p) ! t^{p}}{p !}\left[z^{2 p}\right]\left(1+e^{z}+\cdots+e^{(\ell-1) z}\right) e^{-\frac{(d-1) z}{2}} \times \\
& \times \sum_{j_{\ell}=0}^{n_{\ell}-1} \sum_{j_{\ell+1}=0}^{n_{\ell+1}} \cdots \sum_{j_{q}=0}^{n_{q}} e^{z \sum_{i \geq \ell} i j_{i}}(-1)^{\sum_{i \geq \ell} j_{i}}\left(\begin{array}{c}
n_{\ell}-1 \\
j_{l}
\end{array}\right)\left(\begin{array}{c}
n_{\ell+1} \\
j_{\ell+1}
\end{array}\right) \ldots \\
& \left(\begin{array}{c}
n_{q} \\
j_{q}
\end{array}\right) \ldots(\mathrm{Lemma3.3)} \\
= & \sum_{p=0}^{\infty} \frac{(2 p) ! t^{p}}{p !}\left[z^{2 p}\right] e^{-\frac{(d-1) z}{2}}\left(1+e^{z}+\cdots+e^{(\ell-1) z}\right) \\
& \left(1-e^{\ell z}\right)^{n_{\ell}-1} \prod\left(1-e^{i z}\right)^{n_{i}} \\
= & \sum_{p=0}^{\infty} \frac{(2 p) ! t^{p}}{p !}\left[z^{2 p}\right] e^{-\frac{(d-1) z}{2}} \prod_{i \geq 1}\left(1-e^{i z}\right)^{c_{i}} . \\
& (1 \geq \ell+1
\end{aligned}
$$

Finally we get:

$$
H_{(d), \beta}^{g,(2)}=\frac{s ! d^{s-1}}{2^{s} \prod \beta_{j}}\left[t^{s}\right] \exp \left(\frac{t d^{2}}{12}\right) \sum_{p=0}^{\infty} \frac{(2 p) ! t^{p}}{p !}\left[z^{2 p}\right] e^{-\frac{(d-1) z}{2}} \prod_{i \geq 1}\left(1-e^{i z}\right)^{c_{i}}
$$




$$
\begin{aligned}
& =\frac{s ! d^{s-1}}{2^{s}}\left[t^{s}\right] \exp \left(\frac{t d^{2}}{12}\right) \sum_{p=0}^{\infty} \frac{(2 p) ! t^{p}}{p !}\left[z^{2 p-n+1}\right] \prod_{i \geq 1}\left(\frac{\sinh (i z / 2)}{i z / 2}\right)^{c_{i}} \\
& =\frac{s ! d^{s-1}}{2^{s}} \sum_{h=0}^{s} \frac{(2 s-2 h) !}{h !(s-h) ! 12^{h}} d^{2 h}\left[z^{2 s-2 h-n+1}\right] \prod_{i \geq 1}\left(\frac{\sinh (i z / 2)}{i z / 2}\right)^{c_{i}}
\end{aligned}
$$

To pass from the first to the second line, we write $1-e^{i z}=-2 e^{i z / 2} \sinh (i z / 2)$ and use $\sum_{i} c_{i}=n-1, \sum_{i} i c_{i}=d-1$ and $\prod_{i} i^{c_{i}}=\prod \beta_{j}$. There is also the factor $(-1)^{\sum c_{i}}=(-1)^{n-1}=1$ since $n$ is odd.

Note that $2 s=2 g-1+n$, so we are taking the coefficient of $z^{2(g-h)}$. Because the lowest degree of the series in $z$ is 0 , the summing index $h$ actually runs from 0 to $g$. Finally, we get:

$$
H_{(d), \beta}^{g,(2)}=\frac{s ! d^{s-1}}{2^{s}} \sum_{h=0}^{g} \frac{(2 s-2 h) !}{h !(s-h) ! 12^{h}} d^{2 h}\left[z^{2(g-h)}\right] \prod_{i \geq 1}\left(\frac{\sinh (i z / 2)}{i z / 2}\right)^{c_{i}} .
$$

Using Lemma 3.2, we obtain the second equation of Theorem 1.2.

\section{Some corollaries}

Our formula is explicit and computationally efficient. We observe a strong similarity with the case of ordinary one-part double Hurwitz numbers. Consequently, as in [6], we prove some fairly important implications.

\subsection{Strong polynomiality}

Our formula gives immediately the strong polynomialty of 1-part double Hurwitz numbers with completed 3-cycles. In fact, double Hurwitz numbers with completed cycles of any size satisfy the strong piecewise polynomiality, i.e., they are piecewise polynomial with the highest and lowest orders respectively $(r+1) s+1-m-n$ and $(r+1) s+1-m-n-2 g$. This is proved in [13]. For one-part numbers, piecewise polynomiality becomes polynomiality. Our formula should be viewed as an illustration of this fact through an explicitly computable case.

Corollary 4.1 $H_{(d), \beta}^{g,(2)}$, for fixed $g$ and $n$, is a polynomial of the parts of $\beta$ and satisfies the strong polynomiality property, i.e., it is polynomial in $\beta_{1}, \beta_{2}, \ldots$ with highest and lowest degrees, respectively, $3 g+\frac{n-3}{2}$ and $g+\frac{n-3}{2}$

\subsection{Connection with intersection theory on moduli spaces and "the $\lambda_{g}$ theorem"}

The connected single Hurwitz number $\tilde{H}_{\beta}^{g}$ is defined to be the number of weighted equivalence classes of degree $d$ branched covers of $\mathbb{C P}^{1}$ by a connected Riemann surface of genus $g$, with $p+1$ branch points, of which $p$ are simple, and one has 
branching given by $\beta$. Due to the Riemann-Hurwitz formula, we have $p=2 g-2+$ $d+n$, where $n=l(\beta)$. The celebrated ELSV formula [3] connects these numbers with integrals on the moduli space of stable curves:

$$
\tilde{H}_{\beta}^{g}=C(g, \beta) \int_{\overline{\mathcal{M}}_{g, n}} \frac{1-\lambda_{1}+\lambda_{2}-\cdots+(-1)^{g} \lambda_{g}}{\left(1-\beta_{1} \psi_{1}\right) \ldots\left(1-\beta_{n} \psi_{n}\right)}
$$

where

$$
C(g, \beta)=\frac{p !}{\operatorname{Aut}(\beta)} \prod_{i=1}^{n} \frac{\beta_{i}^{\beta_{i}}}{\beta_{i} !}
$$

and $\overline{\mathcal{M}}_{g, n}$ is the moduli space of stable curves of genus $g$ with $n$ marked points. $\lambda_{i}$ is a certain codimension $i$ cohomology class, and $\psi_{i}$ is a certain codimension 1 cohomology class on $\overline{\mathcal{M}}_{g, n}$. The factors $1 /\left(1-\beta_{i} \psi_{i}\right)$ are understood as

$$
1 /\left(1-\beta_{i} \psi_{i}\right)=1+\beta_{1} \psi_{i}+\beta_{1}^{2} \psi_{i}^{2}+\cdots
$$

Although the series are infinite, by the definition of the integral, only a finite number of terms do contribute. Therefore, Eq. (20) means that $P_{n}^{g}(\beta):=\tilde{H}_{\beta}^{g} / C(g, \beta)$ is polynomial in $\beta_{1}, \ldots, \beta_{n}$ and the (linear) Hodge integrals are given by:

$$
\left\langle\tau_{b_{1}} \ldots \tau_{b_{n}} \lambda_{k}\right\rangle_{g}:=\int_{\overline{\mathcal{M}}_{g, n}} \psi_{1}^{b_{1}} \ldots \psi_{n}^{b_{n}} \lambda_{k}=(-1)^{k}\left[\beta_{1}^{b_{1}} \ldots \beta_{n}^{b_{n}}\right] P_{n}^{g}(\beta)
$$

Another ELSV formula has been found for the so-called orbifold Hurwitz numbers, i.e., double Hurwitz numbers with $\alpha=(a, a, \ldots, a)$ by Johnson, Pandharipande and Tseng [7]. It is an important and challenging problem to find other ELSV formulas. An important clue is the (piecewise) polynomiality of Hurwitz numbers.

In [13], the authors conjecture that for every $r \geq 1$, there exist moduli spaces $X_{g, n}^{(r)}$ of complex dimension $2 g(r+1)+n-1$ such that we have the following ELSV formula:

$$
H_{(d), \beta}^{g,(r)}=\frac{s !}{d} \int_{X_{g, n}} \frac{1-\Lambda_{2}+\Lambda_{4}-\cdots+(-1)^{g} \Lambda_{2 g}}{\left(1-\beta_{1} \Psi_{1}\right) \ldots\left(1-\beta_{n} \Psi_{n}\right)},
$$

where we fix the degrees of the rational cohomology classes $\Lambda_{2 k} \in H^{4 r k}\left(X_{g, n}^{(r)}\right)$ and $\Psi_{i} \in H^{2 r}\left(X_{g, n}^{(r)}\right)$.

A similar conjecture was previously made by Goulden et al. [6] for ordinary double Hurwitz numbers, i.e., the case $r=1$. To support their conjecture, they made a thorough combinatorial study and found many similarity between the "combinatorial Hodge integrals" and the "genuine" ones defined by Eq. (22) . 
Following them, let us define the combinatorial Hodge integral for $b_{1}, \ldots, b_{n} \geq 0$ and $0 \leq k \leq g$ :

$$
\begin{aligned}
\left\langle\left\langle\tau_{b_{1}} \ldots \tau_{b_{n}} \Lambda_{2 k}\right\rangle\right\rangle_{g} & :=(-1)^{k}\left[\beta_{1}^{b_{1}} \ldots \beta_{n}^{b_{n}}\right]\left(d \frac{H_{(d), \beta}^{g,(2)}}{s !}\right) \\
& =(-1)^{k}\left[\beta_{1}^{b_{1}} \ldots \beta_{n}^{b_{n}}\right]\left(d \frac{H_{(d), \beta}^{g,(2)}}{\left(g+\frac{n-1}{2}\right) !}\right) .
\end{aligned}
$$

We use the double brackets just to remind the readers that the conjectural moduli space has not been found, and do not keep the superscript (2) to save space. This "intersection number" vanishes unless $b_{1}+\cdots+b_{n}+2 k=3 g+\frac{n-1}{2}$. The order of this integral is defined to be $b_{1}+\cdots+b_{n}$.

We are going to evaluate the lowest order terms, i.e., the terms with $k=g$. In [5], Faber and Pandharipande proved the $\lambda_{g}$ conjecture:

$$
\left\langle\tau_{b_{1}} \ldots \tau_{b_{n}} \lambda_{g}\right\rangle_{g}=\mathbf{c}_{g}\left(\begin{array}{c}
2 g-3+n \\
b_{1}, \ldots, b_{n}
\end{array}\right) .
$$

By computing $\left\langle\tau_{1}^{2 g-2} \lambda_{g}\right\rangle_{g}$, they found

$$
\mathbf{c}_{g}=\frac{2^{2 g-1}-1}{2^{2 g-1}(2 g) !}\left|B_{2 g}\right|,
$$

where $B_{2 g}$ is a Bernoulli number $\left(B_{0}=1, B_{2}=1 / 6, B_{4}=-1 / 30, B_{6}=1 / 42, \ldots\right)$. In analogy with this theorem, the following combinatorial version is proved in $[6$, Prop. 3.12]: ${ }^{4}$

$$
\left\langle\left\langle\tau_{b_{1}} \ldots \tau_{b_{n}} \Lambda_{2 g}\right\rangle\right\rangle_{g}^{r=1}=\mathbf{c}_{g}\left(\begin{array}{c}
2 g-3+n \\
b_{1}, \ldots, b_{n}
\end{array}\right) .
$$

Here, thanks to Theorem 1.2, we can also easily evaluate $\left\langle\left\langle\tau_{b_{1}} \ldots \tau_{b_{n}} \Lambda_{2 g}\right\rangle\right\rangle_{g}$.

Proposition 4.2 For $b_{1}+\cdots+b_{n}=g+\frac{n-1}{2}$, the lowest combinatorial Hodge integral is given by:

$$
\left\langle\left\langle\tau_{b_{1}} \ldots \tau_{b_{n}} \Lambda_{2 g}\right\rangle\right\rangle_{g}=\left(\begin{array}{c}
g+\frac{n-1}{2} \\
b_{1}, \ldots, b_{n}
\end{array}\right) \mathbf{C}_{g, n},
$$

with

$$
\mathbf{C}_{g, n}=\frac{(2 g+n-1) !\left(2^{2 g-1}-1\right)}{(2 g) !\left(g+\frac{n-1}{2}\right) ! 2^{3 g+\frac{n-3}{2}}}\left|B_{2 g}\right| .
$$

Proof First, we extract the lowest term in the polynomial $d H_{(d), \beta}^{g,(2)} / s$ ! . This means simply taking only the $h=0$ term in the sum (4), and then taking the constant term

\footnotetext{
$\overline{4}$ Their symbol $\langle\langle.\rangle\rangle_{g}^{r=1}$ is defined just like in Eq. (24), with a different normalization [6, Eq. 25].
} 
of $S_{2 \lambda}$, which is $(-1)^{l(\lambda)}$, in the sum over all partitions $\lambda$ of $g$. The result is:

$$
\frac{(2 s) ! d^{s}}{s ! 2^{s+2 g}} \sum_{\lambda \vdash g} \frac{(-1)^{l(\lambda)} \xi_{2 \lambda}}{|\operatorname{Aut}(\lambda)|}=\frac{(2 g+n-1) ! d^{g+\frac{n-1}{2}}}{\left(g+\frac{n-1}{2}\right) ! 2^{3 g+\frac{n-1}{2}}} \sum_{\lambda \vdash g} \frac{(-1)^{l(\lambda)} \xi_{2 \lambda}}{|\operatorname{Aut}(\lambda)|}
$$

Then we compute the coefficient of $\beta_{1}^{b_{1}} \ldots \beta_{n}^{b_{n}}$ to get the lowest combinatorial Hodge integral:

$$
\left\langle\left\langle\tau_{b_{1}} \ldots \tau_{b_{n}} \Lambda_{2 g}\right\rangle\right\rangle_{g}=(-1)^{g}\left(\begin{array}{c}
g+\frac{n-1}{2} \\
b_{1}, \ldots, b_{n}
\end{array}\right) \frac{(2 g+n-1) !}{\left(g+\frac{n-1}{2}\right) ! 2^{3 g+\frac{n-1}{2}}} \sum_{\lambda \vdash g} \frac{(-1)^{l(\lambda)} \xi_{2 \lambda}}{|\operatorname{Aut}(\lambda)|} .
$$

On the other hand, using (3), we compute:

$$
\begin{aligned}
\left\langle\left\langle\tau_{g} \Lambda_{2 g}\right\rangle\right\rangle_{g} & =(-1)^{g}\left[d^{g}\right] \frac{d H_{(d),(d)}}{g !} \\
& =(-1)^{g}\left[d^{g}\right] \frac{d^{g}(2 g) !}{2^{g} g !}\left[z^{2 g}\right] \frac{z / 2}{\sinh z / 2} \frac{\sinh d z / 2}{d z / 2} \\
& =\frac{(2 g) !}{g ! 2^{g}}(-1)^{g}\left[z^{2 g}\right] \frac{z / 2}{\sinh z / 2} \\
& =\frac{2^{2 g-1}-1}{g ! 2^{3 g-1}}\left|B_{2 g}\right| .
\end{aligned}
$$

Comparing (29) and (30), we deduce $\sum_{\lambda \vdash g} \frac{(-1)^{l(\lambda)} \xi_{2 \lambda}}{|\operatorname{Aut}(\lambda)|}$ and get the desired claim.

We observe a strong similarity with the results quoted above.

\subsection{Dilaton and string equations}

Goulden et al. [6, Prop. 3.10] proved that their combinatorial Hodge integrals for ordinary double Hurwitz numbers satisfy the string and dilation equations. Here we prove that the lowest terms satisfy the (modified) string and dilaton equations for every genus $g$. The situation for higher terms is not clear to us.

Proposition 4.3 String equation: For $g \geq 0, n \geq 1, n$ odd, $b_{1}, \ldots, b_{n} \geq 0, b_{1}+\cdots+$ $b_{n}=g+\frac{n+1}{2}$ :

$$
\left\langle\left\langle\tau_{0}^{2} \tau_{b_{1}} \ldots \tau_{b_{n}} \Lambda_{2 g}\right\rangle\right\rangle_{g}=(2 g+n) \sum_{i=1}^{n}\left\langle\left\langle\tau_{b_{1}} \ldots \tau_{b_{i-1}} \tau_{b_{i}-1} \tau_{b_{i+1}} \ldots \tau_{b_{n}} \Lambda_{2 g}\right\rangle\right\rangle_{g}
$$


Dilaton equation: For $g \geq 0, n \geq 1, n$ odd, $b_{1}, \ldots, b_{n} \geq 0, b_{1}+\cdots+b_{n}=g+\frac{n-1}{2}$ (minus here is not a misprint):

$$
\left\langle\left\langle\tau_{0} \tau_{1} \tau_{b_{1}} \ldots \tau_{b_{n}} \Lambda_{2 g}\right\rangle\right\rangle_{g}=(2 g+n)\left(g+\frac{n+1}{2}\right)\left\langle\left\langle\tau_{b_{1}} \ldots \tau_{b_{n}} \Lambda_{2 g}\right\rangle\right\rangle_{g} .
$$

Here we assume that $\langle\langle\rangle\rangle=$.0 if there is some $\tau_{<0}$ inside.

Proof For the string equation:

$$
\begin{aligned}
\left\langle\left\langle\tau_{0}^{2} \tau_{b_{1}} \ldots \tau_{b_{n}} \Lambda_{2 g}\right\rangle\right\rangle_{g} & =\left(\begin{array}{c}
g+\frac{n+1}{2} \\
b_{1}, \ldots, b_{n}
\end{array}\right) \mathbf{C}_{g, n+1} \\
& =\frac{\left(g+\frac{n-1}{2}\right) !\left(b_{1}+\cdots+b_{n}\right)}{b_{1} ! \ldots b_{n} !} \mathbf{C}_{g, n} \frac{(2 g+n+1)(2 g+n)}{2\left(g+\frac{n+1}{2}\right)} \\
& =(2 g+n) \sum_{i=1}^{n}\left\langle\left\langle\tau_{b_{1}} \ldots \tau_{b_{i-1}} \tau_{b_{i}-1} \tau_{b_{i+1}} \ldots \tau_{b_{n}} \Lambda_{2 g}\right\rangle\right\rangle_{g} .
\end{aligned}
$$

For the dilaton equation:

$$
\begin{aligned}
\left\langle\left\langle\tau_{0} \tau_{1} \tau_{b_{1}} \ldots \tau_{b_{n}} \Lambda_{2 g}\right\rangle\right\rangle_{g} & =\left(\begin{array}{c}
g+\frac{n+1}{2} \\
0,1, b_{1}, \ldots, b_{2 s+1}
\end{array}\right) \mathbf{C}_{g, n+1} \\
& =\left(\begin{array}{c}
g+\frac{n-1}{2} \\
b_{1}, \ldots, b_{n}
\end{array}\right) \mathbf{C}_{g, n}(2 g+n)\left(g+\frac{n+1}{2}\right) \\
& =(2 g+n)\left(g+\frac{n+1}{2}\right)\left\langle\left\langle\tau_{b_{1}} \ldots \tau_{b_{n}} \Lambda_{2 g}\right\rangle\right\rangle_{g} .
\end{aligned}
$$

Let us consider the following generating function:

$$
F:=\sum_{n \geq 1} \frac{1}{n !} \sum_{b_{1}, \ldots, b_{n} \geq 0}\left\langle\left\langle\tau_{b_{1}} \ldots \tau_{b_{n}} \Lambda_{2 g}\right\rangle\right\rangle_{g} \frac{t_{b_{1}} \ldots t_{b_{n}}}{(2 g+n-2) ! !}
$$

Then the string and dilaton equations can be written as follows:

$$
\begin{array}{r}
\left(-\frac{\partial^{2}}{\partial t_{0}^{2}}+\sum_{i=0}^{\infty} t_{i+1} \frac{\partial}{\partial t_{i}}\right) F:=L_{-1} F=0 \\
\left(-\frac{\partial^{2}}{\partial t_{0} \partial t_{1}}+1+\sum_{i=0}^{\infty} i t_{i} \frac{\partial}{\partial t_{i}}\right) F:=L_{0} F=0
\end{array}
$$

It is easy to check that $\left[L_{0}, L_{-1}\right]=L_{-1}$. They look like two lowest Virasoro constraints. It would be interesting to investigate whether we have higher Virasoro-like constraints as well. And of course, it would be of great interest to investigate string and dilaton equations for higher order integrals, i.e., for $\Lambda_{2 k}$ with $k<g$. 


\subsection{Explicit formulae for top degree terms}

We show how to compute the top degree terms $\left\langle\left\langle\tau_{b_{1}} \ldots \tau_{b_{n}}\right\rangle\right\rangle_{g}:=\left\langle\left\langle\tau_{b_{1}} \ldots \tau_{b_{n}} \Lambda_{0}\right\rangle\right\rangle_{g}$. We need to prepare some notation. For a partition $\mu$, let $P_{\mu}$ be the power sum and $m_{\mu}$ be the monomial symmetric function. For any partition $\lambda$, we have the following expansion (in the vector space of symmetric polynomials)

$$
P_{\lambda}=\sum_{\mu \vdash|\lambda|} R_{\lambda \mu} m_{\mu}
$$

One can show that $R_{\lambda \mu}$ is the number of ordered partitions ${ }^{5} \pi=\left(A_{1}, \ldots, A_{l(\mu)}\right)$ of the set $\{1, \ldots, l(\lambda)\}$ such that for $1 \leq j \leq l(\mu)$ :

$$
\mu_{j}=\sum_{i \in A_{j}} \lambda_{i}
$$

For $2 j \leq b_{1}+\cdots+b_{n}$, denote

$$
D_{2 j}(\mathbf{b}):=\left\{\left(a_{1}, \ldots, a_{n}\right), a_{i} \text { even, } a_{i} \leq b_{i}, a_{1}+\cdots+a_{n}=2 j\right\} .
$$

For a vector $\mathbf{a}$, denote $P_{\mathbf{a}}$ the associated partition, i.e., the rearrangement of the components of $\mathbf{a}$ in non-decreasing order.

Proposition 4.4 For $b_{1}, \ldots, b_{n} \geq 0, b_{1}+\cdots+b_{n}=3 g+\frac{n-1}{2}$, we have:

$$
\begin{aligned}
\left\langle\left\langle\tau_{b_{1}} \ldots \tau_{b_{n}}\right\rangle\right\rangle_{g}= & \frac{1}{2^{3 g+\frac{n-1}{2}}} \sum_{h=0}^{g} \frac{(2 s-2 h) !}{h !(s-h) ! 3^{h}} \sum_{\lambda \vdash(g-h)} \sum_{\mathbf{a} \in D_{2 g-2 h}(\mathbf{b})} \\
& \frac{\xi_{2 \lambda} R_{2 \lambda, P_{\mathbf{a}}}}{|A u t(\lambda)|}\left(\begin{array}{c}
g+\frac{n-1}{2}+2 h \\
b_{1}-a_{1}, \ldots, b_{n}-a_{n}
\end{array}\right) .
\end{aligned}
$$

Proof First, we need to extract the highest degree term in $d H_{(d), \beta}^{g} / s$ !. The result is:

$$
T T_{n}^{g}=\frac{d^{s}}{2^{s+2 g}} \sum_{h=0}^{g} \frac{(2 s-2 h) !}{h !(s-h) ! 3^{h}} d^{2 h} \sum_{\lambda \vdash(g-h)} \frac{\xi_{2 \lambda} P_{2 \lambda}}{|\operatorname{Aut}(\lambda)|},
$$

Then as usual, we compute the coefficient of $\beta_{1}^{b_{1}} \ldots \beta_{n}^{b_{n}}$ to obtain $\left\langle\left\langle\tau_{b_{1}} \ldots \tau_{b_{n}}\right\rangle\right\rangle_{g}$. Using the obvious fact that $\left[x_{1}^{d_{1}} x_{2}^{d_{2}} \ldots\right] m_{\mu}(x)=1$ if $\mu=P_{\mathbf{d}}$ and 0 otherwise, we obtain the desired claim.

In particular, for $g=1$ :

\footnotetext{
5 Do not confuse partitions of a number and partitions of a set.
} 
Corollary 4.5 For $n \geq 1, b_{1}, \ldots, b_{n} \geq 0$ and $b_{1}+\cdots+b_{n}=\frac{n+5}{2}$ :

$$
\begin{aligned}
\left\langle\left\langle\tau_{b_{1}} \ldots \tau_{b_{n}}\right\rangle\right\rangle_{1}= & \frac{(n+1) !}{3 \times 2^{\frac{n+7}{2}}}\left[\frac{1}{n}\left(\begin{array}{c}
\frac{n+5}{2} \\
b_{1}, \ldots, b_{n}
\end{array}\right)\right. \\
& \left.+\sum_{i=1}^{n}\left(\begin{array}{c}
\frac{n+1}{2} \\
b_{1}, \ldots, b_{i-1}, b_{i}-2, b_{i+1}, \ldots, b_{n}
\end{array}\right)\right],
\end{aligned}
$$

where $\left(\begin{array}{l}\frac{n+1}{2} \\ b_{1}, \ldots, b_{i-1}, b_{i}-2, b_{i+1}, \ldots, b_{n}\end{array}\right)=0$ if $b_{i}-2<0$.

One can compare this formula with the Hodge integrals over $\overline{\mathcal{M}}_{1, n}$ which can be found, for instance, in [10, Prop. 4.6.11]:

Proposition 4.6 For $d_{1}+\cdots d_{n}=n$, we have:

$$
\begin{aligned}
\left\langle\tau_{d_{1}} \ldots \tau_{d_{n}}\right\rangle & :=\int_{\overline{\mathcal{M}}_{g, n}} \psi_{1}^{d_{1}} \ldots \psi_{n}^{d_{n}} \\
& =\frac{1}{24}\left(\begin{array}{c}
n \\
d_{1} \ldots d_{n}
\end{array}\right)\left(1-\sum_{i=2}^{n} \frac{(i-2) !(n-i) !}{n !} e_{i}\left(d_{1}, \ldots, d_{n}\right)\right),
\end{aligned}
$$

where $e_{i}$ is the ith elementary symmetric function:

$$
e_{i}\left(d_{1}, \ldots, d_{n}\right)=\sum_{j_{1}<\ldots<j_{i}} d_{j_{1}} \ldots d_{j_{i}} .
$$

Acknowledgements This article is a part of my Ph.D. thesis that I am preparing under the supervision of Mattia Cafasso and Vladimir Roubtsov at LAREMA, UMR CNRS 6093. I am grateful to my supervisors for continuous support. I am also grateful to Bertrand Eynard and Vincent Rivasseau for having guided my first steps in research with extreme care during my research internship. This experience played a key role in my decision to continue the hard path of research. My Ph.D. study is funded by the French ministerial scholarship "Allocations Spécifiques Polytechniciens". My research is also partially supported by LAREMA and the Nouvelle Équipe "Topologie algébrique et Physique Mathématique" of the Pays de la Loire region.

\section{References}

1. Alexandrov, A.: Enumerative geometry, tau-functions and Heisenberg-Virasoro algebra. Comm. Math. Phys. 338(1), 195-249 (2015)

2. Cavalieri, R., Johnson, P., Markwig, H.: Tropical Hurwitz numbers. J. Algebraic Combin. 32(2), 241265 (2010)

3. Ekedahl, T., Lando, S., Shapiro, M., Vainshtein, A.: Hurwitz numbers and intersections on moduli spaces of curves. Invent. Math. 146(2), 297-327 (2001)

4. Eynard, B., Mulase, M., Safnuk, B.: The Laplace transform of the cut-and-join equation and the Bouchard-Mariño conjecture on Hurwitz numbers. Publ. Res. Inst. Math. Sci. 47(2), 629-670 (2011)

5. Faber, C., Pandharipande, R.: Hodge integrals, partition matrices, and the $\lambda_{g}$ conjecture. Ann. Math. (2) 157(1), 97-124 (2003)

6. Goulden, I.P., Jackson, D.M., Vakil, R.: Towards the geometry of double Hurwitz numbers. Adv. Math. 198(1), 43-92 (2005) 
7. Johnson, P., Pandharipande, R., Tseng, H.-H.: Abelian Hurwitz-Hodge integrals. Michigan Math. J. 60(1), 171-198, 04 (2011)

8. Kazarian, M.E., Lando, S.K.: Combinatorial solutions to integrable hierarchies. Russian Math. Surveys 70(3), 453 (2015)

9. Kerov, S., Olshanski, G.: Polynomial functions on the set of Young diagrams. C. R. Acad. Sci. Paris Sér. I Math. 319(2), 121-126 (1994)

10. Lando, S.K., Zvonkin, A.K.: Graphs on Surfaces and Their Applications. Springer, Berlin (2004)

11. Okounkov, A.: Toda equations for Hurwitz numbers. Math. Res. Lett. 7(4), 447-453 (2000)

12. Okounkov, A., Pandharipande, R.: Gromov-Witten theory, Hurwitz theory, and completed cycles. Ann. Math. (2) 163(2), 517-560 (2006)

13. Shadrin, S., Spitz, L., Zvonkine, D.: On double Hurwitz numbers with completed cycles. J. Lond. Math. Soc. (2) 86(2), 407-432 (2012)

14. Shadrin, S., Spitz, L., Zvonkine, D.: Equivalence of ELSV and Bouchard-Mariño conjectures for $r$-spin Hurwitz numbers. Math. Ann. 361(3-4), 611-645 (2015) 